\title{
Centchroman versus Tamoxifen in the management of Mastalgia: A Randomized Controlled Clinical Trial
}

Khadka S, Rajbanshi S,Khaniya S, Agrawal C.S, Adhikary S.

Department of Surgery, B.P Koirala Institute of Health Sciences, Dharan

Correspondence: Dr Sarada Khadka, Senior Resident,Department of Surgery

Email: kdkadrsarada@gmail.com

\begin{abstract}
Introduction and Objective: Mastalgia is defined as painful nodularity for more than 1 week of menstrual cycle. The long list of drugs highlights the ongoing debate about the drug of choice for the management of mastalgia. So in this study, we compared the effectiveness and cost of Centchroman and Tamoxifen in the management of mastalgia.
\end{abstract}

Materials and methods: A total of 106 female patients with the clinical diagnosis of mastalgia were enrolled in the study and randomized to 1:1 ratio into Centchroman and Tamoxifen group for a period of 1 year. The duration of therapy was 3 months. All patients completed the study and follow up. The response of therapy was assessed on 1, 2, 3 and 6 months by using a Visual Analogue Scale (VAS).

Results: Baseline mean VAS score was 6.25 in Tamoxifen and 6.49 in Centchroman group.There was marked improvement in VAS score after the treatment in both the groups, which was statistically significant with greater reduction in Centchroman group $(p=0.001)$.The number of patients who achieved VAS less than 3 were 51(96.2\%) in Centchroman and 49(92.5\%) in Tamoxifen group at the end of treatment. Tamoxifen was found to be statistically significant and cost-effective $(p<0.001)$ in comparison to Centchroman.

Conclusion: We conclude that Centchroman is not inferior to Tamoxifen and both the drugs effectively reduced pain, however Centchroman reduced the pain more than Tamoxifen.

Key words: Centchroman, Mastalgia, Tamoxifen, VAS 